LA-UR $-91-987$

$$
\begin{gathered}
\text { RE: nST! } \\
\text { AP:O } 1999
\end{gathered}
$$

TITLE: The A Dependence of Dilepton Production

AUTHOR(s): Gerald T. Garvey

Sunmitted to: The Proceedings of the Second E:uropeian Workshop on lladronic Physics with Electrons Beyond 10 GeV

Dourdam, 8-12 Octole $\cdot 190)$.

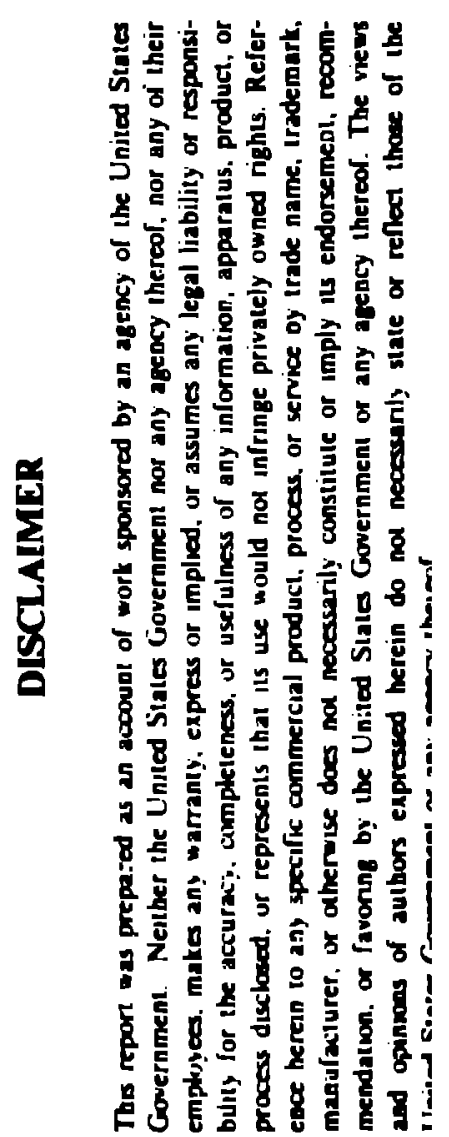

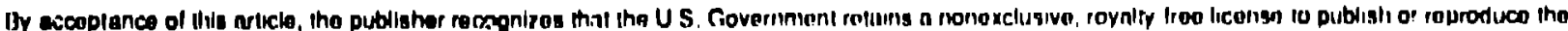
publiahod lorm of dive coninbulion, or to allow othors lo do no, lor U S. Govomment purposes. 


\section{The A dependence of dilepton production}

\section{G. T. Garvey}

Los Alamos National Laboratory, MS H846, Los Alamos, New Mexico 87545, U.S.A.

\section{INTRODUCTION}

The discovery [1] in 1982, by the EMC group, that the structure function $F_{2}(x)$ per nucleon is different in iron than deuterium was the first evidence that the structure of the nucleon might be altered in nuclei. Much more extensive and precise data have been taken since the original discovery. This body of data is now quite consistent [2], and many of the features of the original "EMC effect" remain intact. There have been a variety of theoretical explanations of the data, but none are totally compelling. The deep-inelastic-scattering (DIS) data for the ratio of $F_{2}^{\mathrm{Fe}}(x)$ in iron to $F_{2}^{\mathrm{D}}(x)$ in deuterium are shown in Figure 1 for the region $0.03 \leq x \leq \sim 1.0$. Over this region in

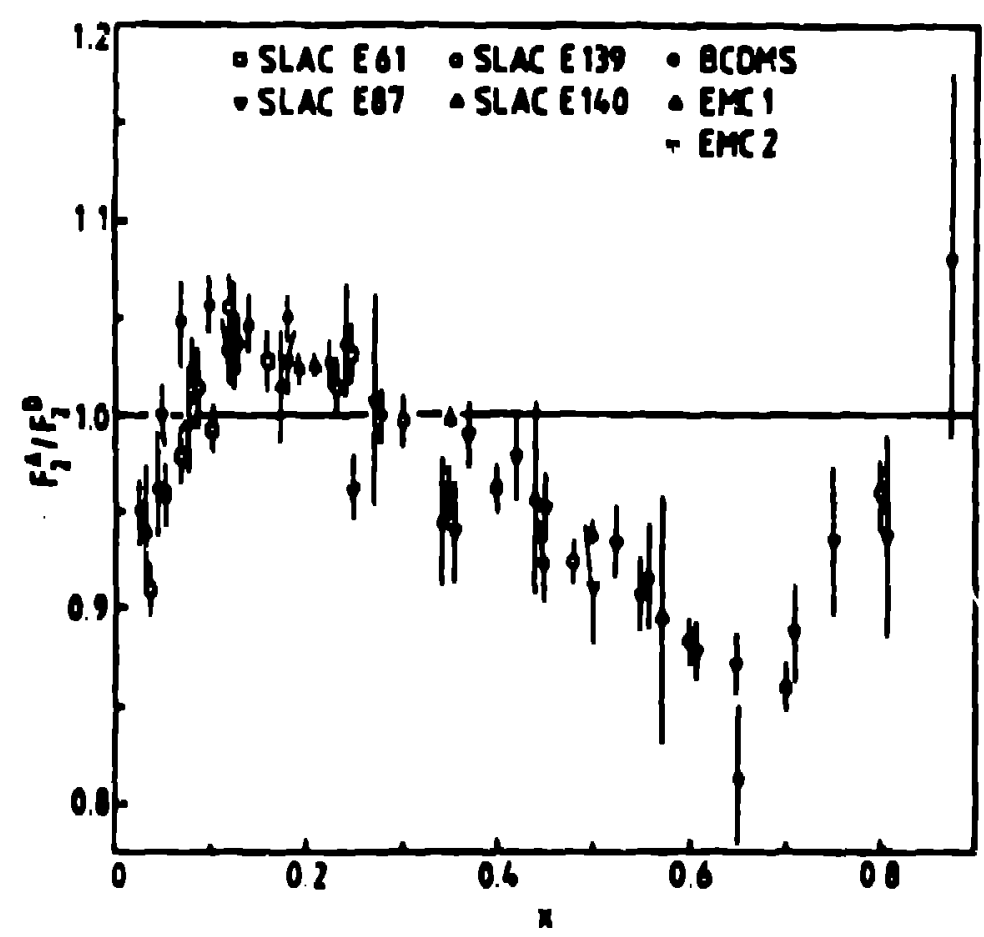

Figure 1. The ratio of the st ructure function $F_{2}(r)$ per mueleron mensured in iron (Fr) nud drutrium (D). The dnten nere a compilntion of severnl mensurements. 
$x$, both suppression and enhancement is observed. Given the fact that there is no way of reliably calculating the structure function for a free nucleon, it is not surprising that there is no satisfactory explanation of th small (10-20\%) alterations that take place in nuclej. All the suppressions and enhan zements, however, appear to be quite regular and increase as a function of $A$. To discuss these effects, it is useful to distinguish four regions. All effects increase as a function of $A$ with the ratio $F_{2}^{A}(x) / F_{2}^{D}(x)$ approaching 1 for all $x$ as $\mathrm{A} \rightarrow 2$. The effects to be noted are: (1) apparent shadowing in the region $x<0.1$; (2) enhancement in the region $0.1 \leq x \leq 0.3$; (3) decrease of the ratio between $0.3 \leq x \leq \sim 0.65$; (4) followed by an increase in the region $\sim 6.5 \leq x \leq 1$. This latter rise is ascribed to Fermi motion of the nucleons within a nucleus and appears to be accounted for by relatively strughtforward calculation.

\section{THE DREI.I,YAN PROCESS}

In order to provide additional information on the EMC effect, our collaboration [3] (FNAL E772) usdertook a measurement of the ratio of the sea quark structure function in nuclei relative to that in deuterium, i.e., $R(A / D)=\bar{g}^{A}(x) / \bar{q}^{D}(x)$. This study was carried out usaig the Dreil-Yan [4] process. If all of the "EMC cifects" at. $0.03 \leq x \leq 0.4$ are ascribable to the sea cuarks, the alteration in the ratio $R(A / D)$ would be very dramatic, as shown in Figure 2. There exist explicit models of the EMC

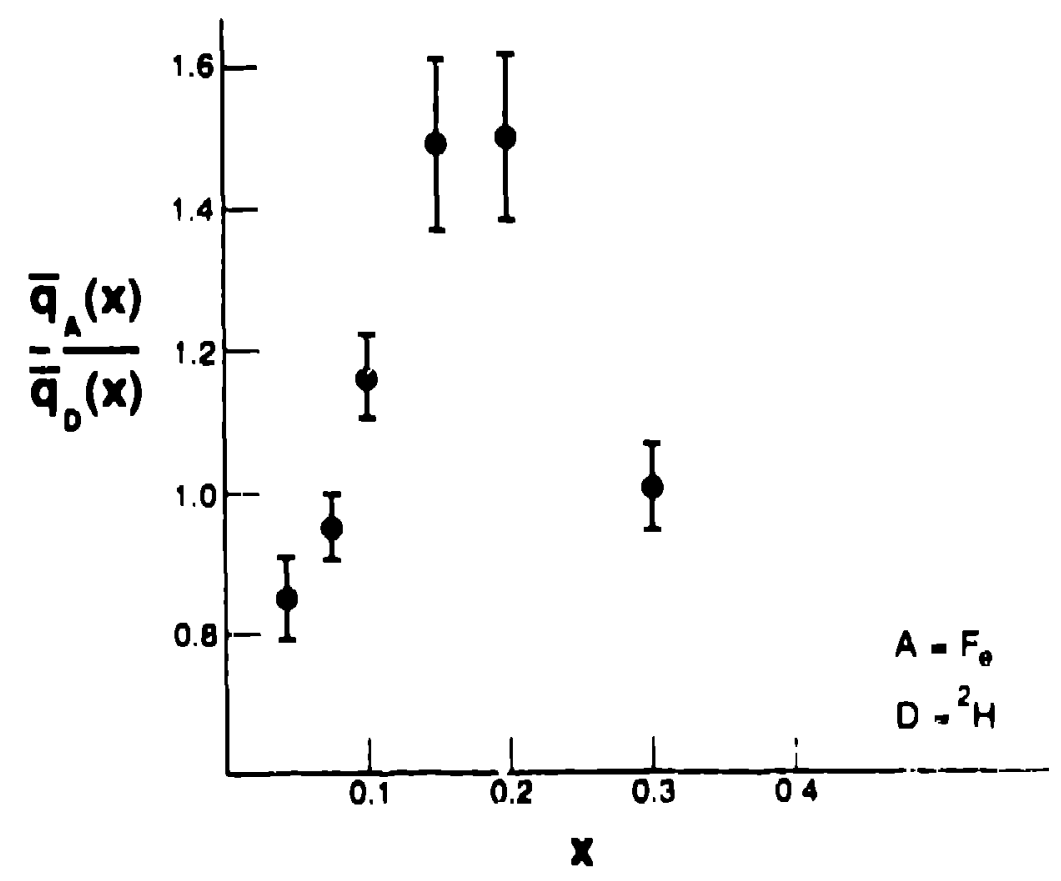

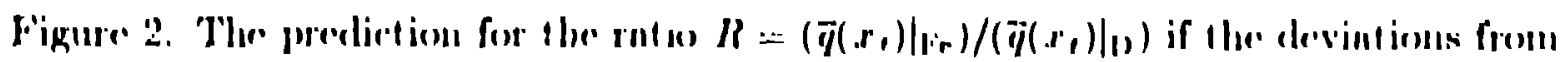

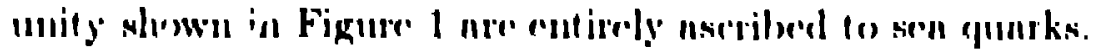


effect that would predict $R(\mathrm{~A} / \mathrm{D})$ to be large, for example, the pion excess model [5] in which momentum is transferred from valence quarks to the sea via a pionic excess generated in finitc nuclei. The idea of using the Drell-Yan process to access the sea quark distribution came from a variety of authors [6], all seeking additional information to interpret the EMC effect.

The lowest-order diagram for the Drell-Yan process is shown in Figure 3. In this case, a quark (antiquark) from the beam projecti.e annihilates in an antiquark (quark) from the target, forming a virtual photon of mass $M$, which in turn decays into a dilepton pair $\left(\ell^{+} \ell^{-}\right)$. The cross section for this process can be written

$\frac{d \sigma}{d M d x_{F}}=\frac{K 8 \pi \alpha^{2}}{g M^{3}} \sum \frac{e_{i}^{2}\left(x_{b} x_{t}\right)}{x_{b}+x_{t}}\left[g_{i}\left(x_{b}\right) \bar{q}_{i}\left(x_{t}\right)+\bar{r}_{i}\left(x_{b}\right) q_{i}\left(x_{t}\right)\right]$,

where $e_{i}$ is the charge of a quark with flavor $i$, and $q_{i}\left(x_{b}\right)$ is the probability that a beam quark of flavor $i$ carries a momentum fraction $\left(x_{b}\right)$, similarly for the target quarks $\left(x_{i}\right)$. 'The factor $K^{\prime}$ is present to account for all higher-order QCD interactions not adsorbed into the definition of the quark structure functions as determined in deep inelastic scat tering.

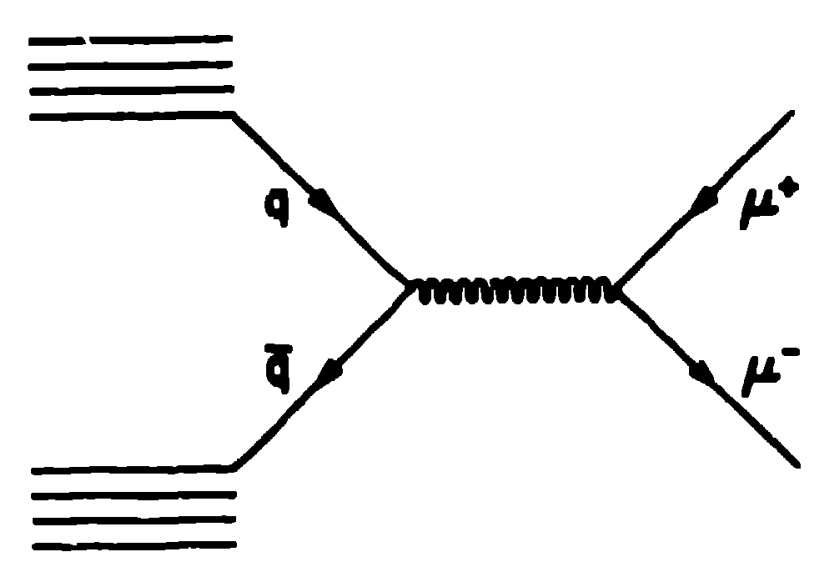

Figure 3. Dingrnm of the Drell-Y'un process.

As we are interested in the bellunvior of the struct une functions reintive to these

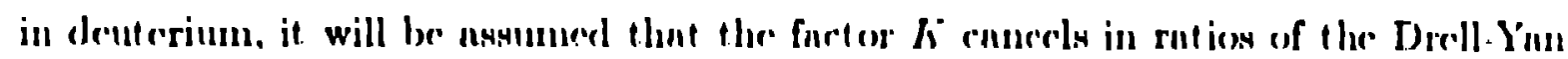

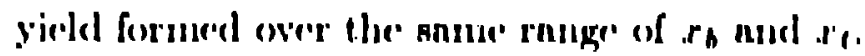

The clunsk structure funetions for the proton ner now rnt here well known. Thery

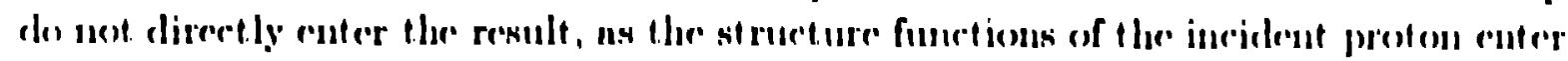

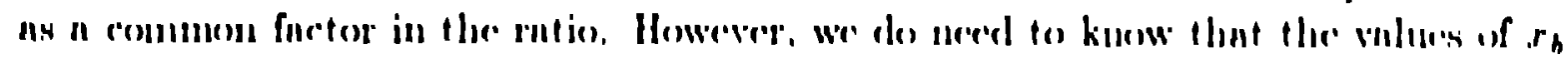

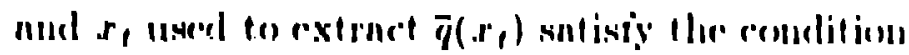

$\eta\left(x_{b}\right) \bar{q}\left(r_{1}\right)>\bar{T}\left(r_{b}\right) q\left(r_{t}\right)$ 
With the above, the relative sea quark distribution in the target nucleus is extracted. For an incident proton, the ratio when $x_{b}>x_{t}$, i.e., $x_{F}>0.2$, of the Drell-Yan yield is therefore

$\left.\frac{\frac{d \sigma}{d M d x_{F}}}{\frac{d \sigma}{d M d x_{F}}}\right|^{\wedge} \frac{2}{A}=\frac{4 u\left(x_{p}\right) \bar{u}^{\wedge}\left(x_{\ell}\right)+d\left(x_{p}\right) \bar{d}^{A}\left(x_{\ell}\right)}{4 u\left(x_{p}\right) \bar{u}^{D}\left(x_{t}\right)+d\left(x_{p}\right) \bar{d}^{D}\left(x_{\ell}\right)}$,

which, in the limit that $x_{p} \rightarrow 1$, gives the simple result that

$\left.\frac{\left.\frac{d \sigma}{d M d x_{F}}\right|^{\mathrm{A}}}{\frac{d \sigma}{d M d x_{F}}}\right|^{\mathrm{D}} \frac{2}{A} \underset{x_{p}-1}{\longrightarrow} \frac{\bar{u}^{A}\left(x_{\ell}\right)}{\bar{u}^{\mathrm{D}}\left(x_{\ell}\right)} \simeq \frac{s^{A}\left(x_{\ell}\right)}{s^{\mathrm{D}}\left(x_{\imath}\right)}$

assuming that the sea and $\bar{u}$ distributions are the same.

The kinematice of the Drell-Yan process is straiglitforward and learls to the following results in the center of mass,

$M^{2}=S x_{b} x_{i}$,

where $S$ is the squared center-of-mass energy

$S \cong 2 M_{N} E_{B}$

$M_{N}$ is the nucleon mass, and $E_{B}$ is the bean chergy. In the lab system wr have

$M^{2}=\left(p_{\ell}+p_{\ell-}\right)^{2} \cong p_{\ell+} p_{\ell-} \theta^{2}$,

where $\theta$ is the Inboratory angle betwern the dileptons and $p_{e \pm}$ is their moment unn. The totnl energy in the lnb system of the Irpton pair is

$E_{l^{+}}+E_{r . .}=E_{p, r_{b}}$

where $E_{p}$ is the laborentory energy of the incident proton nond

$E_{p^{+}}-E_{\ell^{-}}=E_{p} \cdot \operatorname{rscos} \theta_{1}$.m..

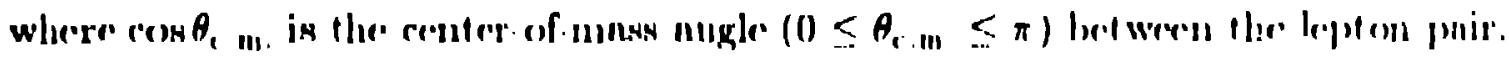




\section{THE EXPERIMENT}

The experiment was carried out using the $800-\mathrm{MeV}$ external proton beam at FNAL. It uses the double-bend dilepton spectrometer previously used in E605 [7]. The targets amployed are ${ }^{2} \mathrm{H}_{2},{ }^{12} \mathrm{C},{ }^{10} \mathrm{Ca}, \mathrm{Fe}$, and $\mathrm{W}$. Each is carefully prepared to minimize any systematic error in relative yield and they are frequently interchanged in the course of a run.

The incident beam current is limited to $2 \times 10^{12}$ per 20 -s spill to keep nultiple hits in the wire chambers below a rate that would introduce ambiguity into the tracking. The angular acceptance of the spectrometer system allows an approximately $5 \%$ acceptance for Drell-Yan events. Figure 4 shows the $x_{b}$ and $x_{t}$ distributions, while
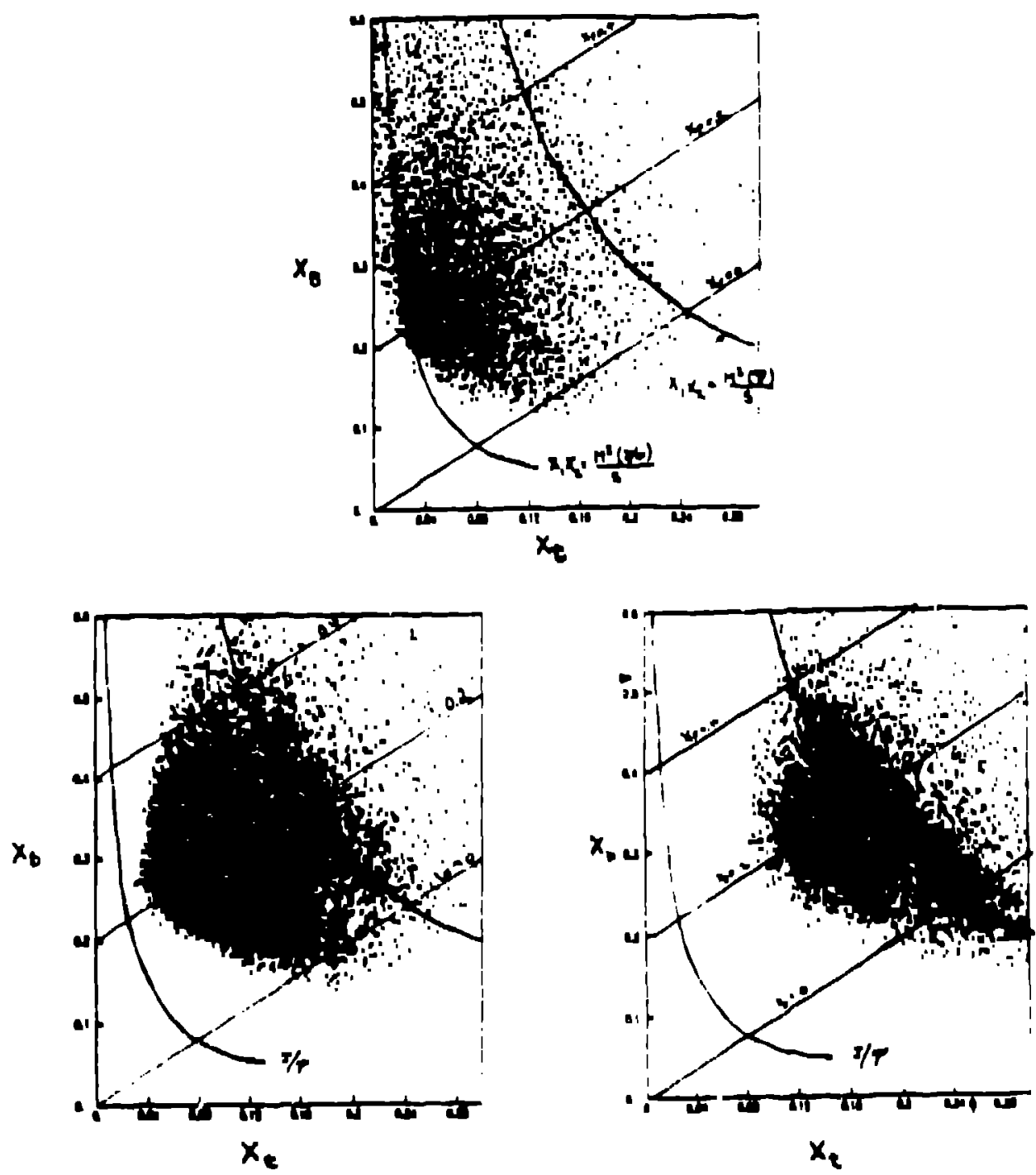

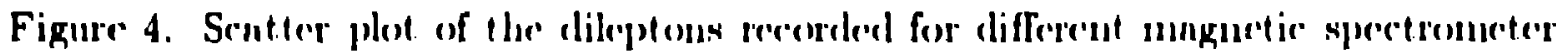
settings ns $n$ function of $r_{n}$ nnel $r_{1}$. 
Figure 5 shows the acceptance-currerted mass spectrum obtained for the three settings of the spectrometer. The overall resolution is the order of $100 \mathrm{MeV}$ and the dimuon pairs from the decay of $J / \psi, \psi^{\prime}$, and $\Upsilon(1 s)$ : $\Upsilon(s 2), \Upsilon(3 s)$ are all well resolved from the underlying Drell-Yan continuum.

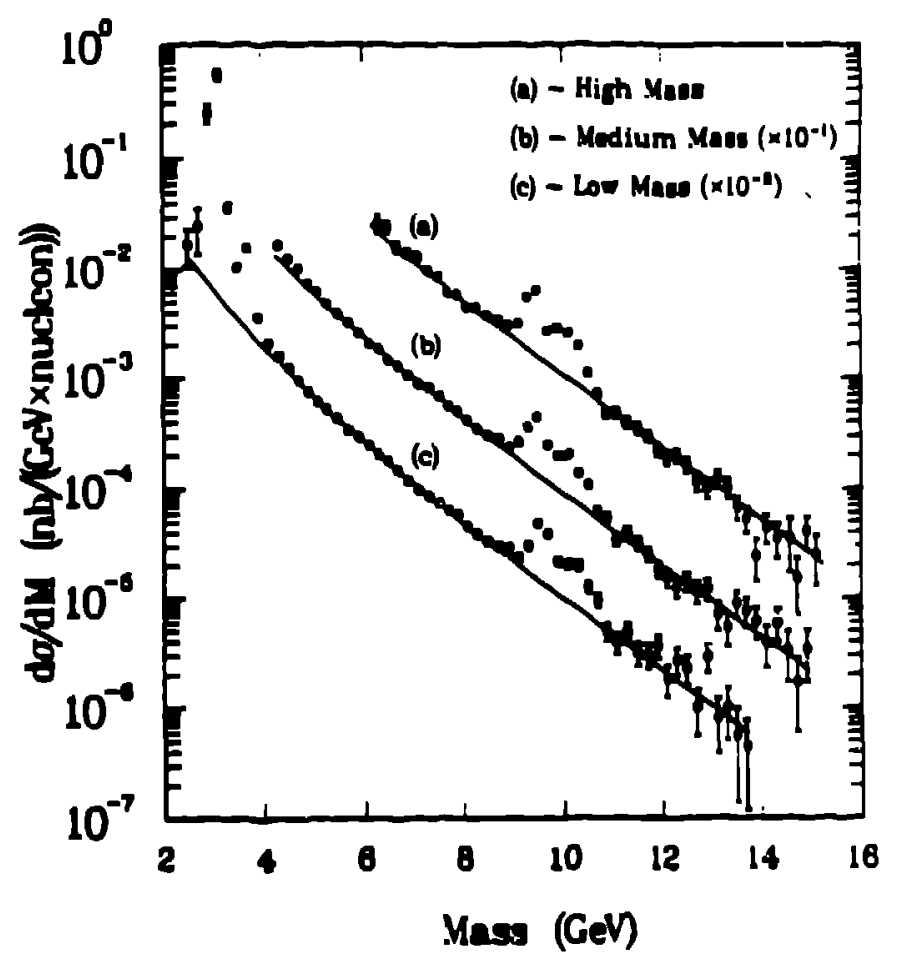

Figure 5. The acceptance-corrected mass spectrum $\left[M=\left(S r_{b} x_{l}\right)^{1 / 2}\right]$ from ench of the spectrometei settings.

\section{RESULTS}

The first olservation to be made is that the measured $A$ dependence of the integrated observed Drell-Yan yield over the range $0.05 \leq x_{1} \leq 0.3,4.2<\mathrm{M}_{\mu}+\mu-$ $12 \mathrm{GrV}$ is very weak. Parnmercrizing the relntive yield as

$\frac{Y_{D Y Y}(A)}{Y_{D Y}(Z)}=$ const $A^{m \text { WY }}$.

we find $n$ oy $=0.098 \pm 0.001$. This result (shown in Figure 0 ) is mush more precise thun the previous experiments nnel supporte the iclens of severnl theorists [8]. that the yirle] of hard processes such an DY is A independent. Thus, even though the soft purions in the proton are grently altereel, the longitudimal monentum of the hurel (valenere) gumrke $\left(s_{b}>0.2\right)$ is only slightly nltered. This gives grently incrensed confidenere thent 


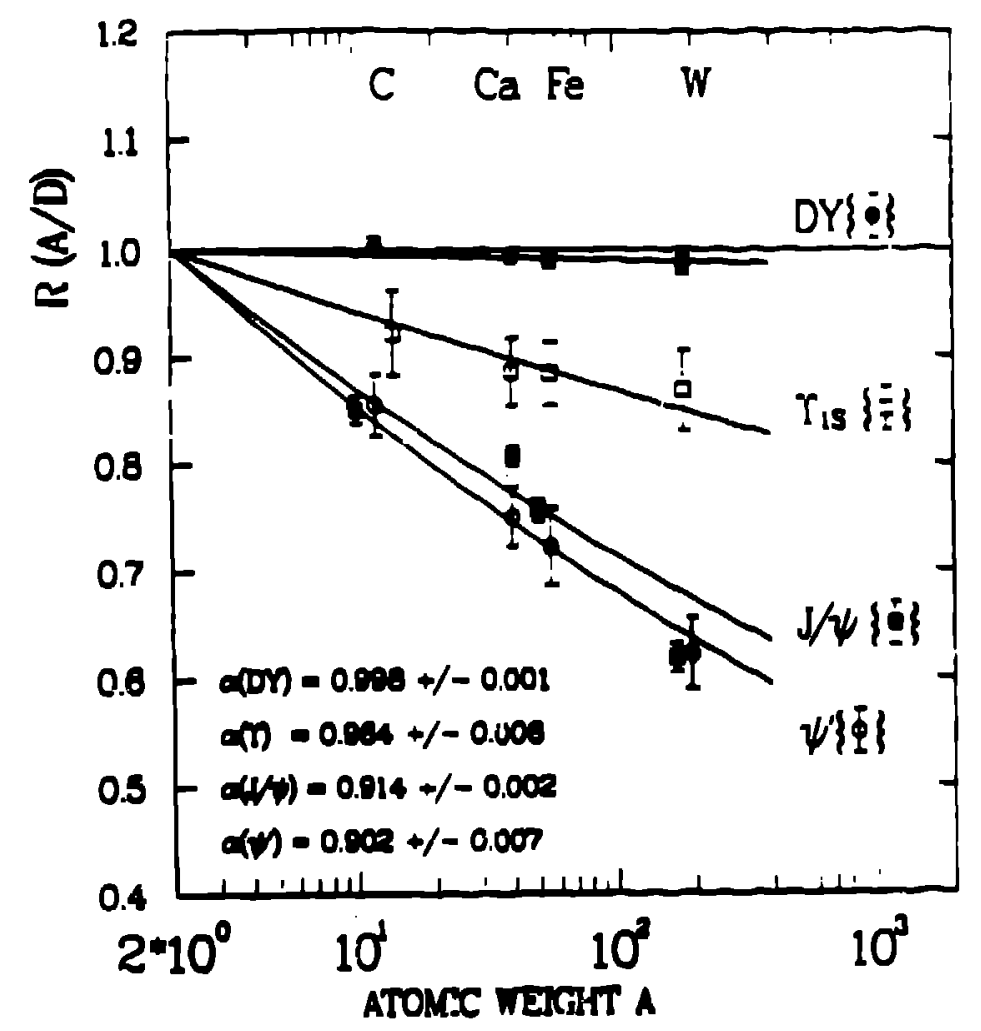

Figure 6. The A dependence of the yield per nucleon for various processes (DY, $J / \psi$ production, $\Upsilon$ production) that produce $\mu_{+} \mu_{-}$pairs. The yield of each process $p$ is parameterized as $Y_{p}(\mathrm{~A})=C_{p} A^{\alpha_{p}}$.

the underlying structure functions of the target are being measured. Figure 7 shows the results for the ratio of the yield of $\mathrm{Fe} /{ }^{2} \mathrm{H}$, along with earlier results [9] achieved with neutrino deep inelastic scattering. There is obviously an enormnus improvement in the duality of the data, which slows very little, if any, enhancemenl in the relative number of sea quarks per nucleon in Fe relntive to those present in ${ }^{2} \mathrm{H}$. One can see that there is evidence for a "shadowing" sffect for $x<0.1$. The small depletion at $x<0.1$ is consistently seen in all our data, and it iscreases with $A$, just as in DIS and, further. there is good agrecment as to its magnitude between our measurements and those in DIS. This is surprising, as a virtual photon in Drell-Yan is time-like and that of DIS is space-like. If shadowing, i.c., the attenuation of the virtual photon, is not the cxplariation, then the observed depletion must come from a relative reduction of the structure function at. low $x$. Figure 8 shows all the Eite duta for the ratio of the Drell-Yan yields from $C\left(\right.$ (a). Ca (b). Fo (c), and W (d) relutive to ${ }^{2} \mathrm{H}$. Figure $7(c)$ shows the Fr $/{ }^{2} \mathrm{H}$ ratio compared to three theories inspired by the EMC effect. The pion excess model [5] predieted nn increase in the number of pione per unclemen in hesvier nuclei clue 


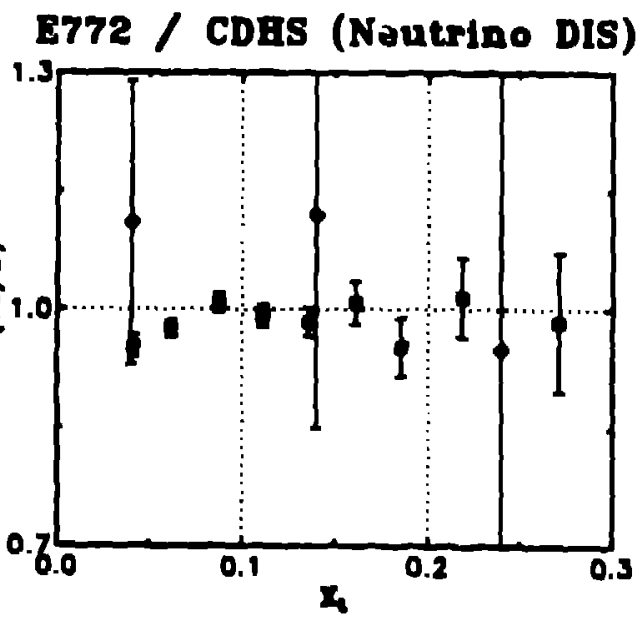

Figure 7. The ratio $R(\mathrm{Fe} / \mathrm{D}) \equiv\left(\left.\bar{q}\left(x_{\ell}\right)\right|_{\mathrm{Fe}}\right) /\left(\left.\bar{q}\left(x_{t}\right)\right|_{\mathrm{D}}\right)$, as determined in this experiment (squares) and previous [9] neutrino DIS (diamonds).

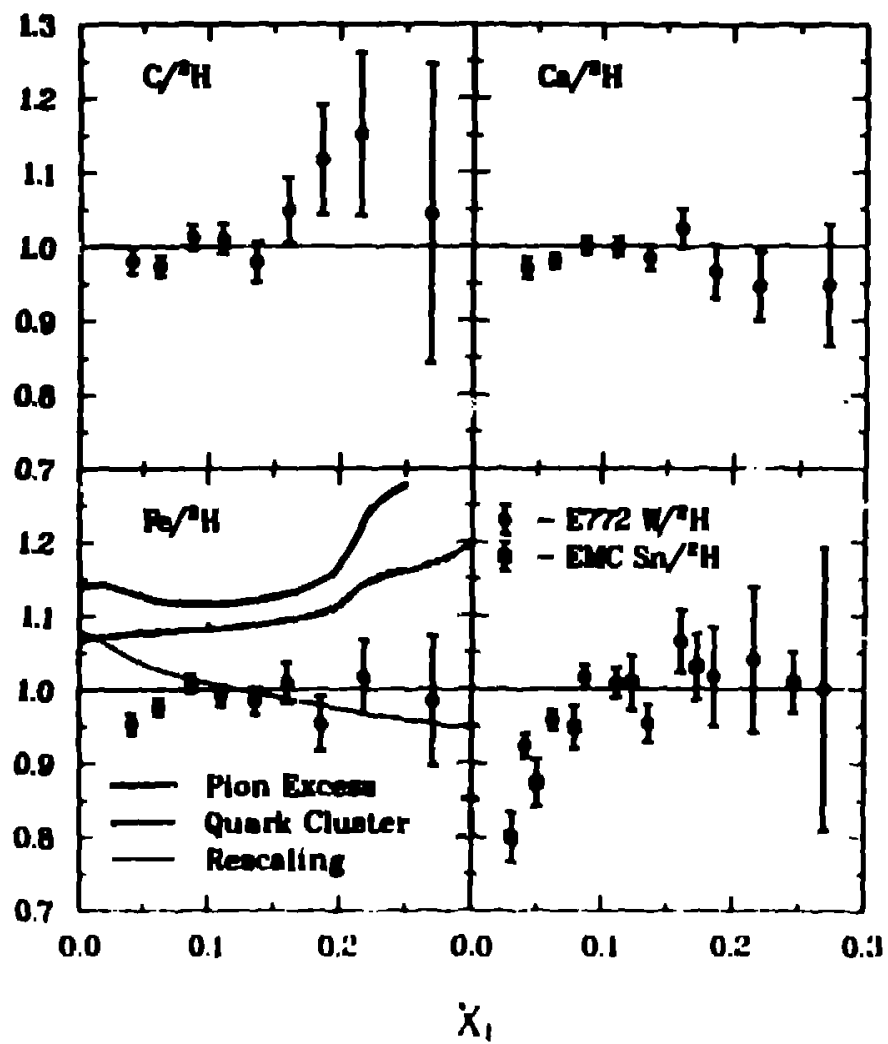

Figure 8. Ratio $\left(\left.\bar{q}\left(x_{1}\right)\right|_{A}\right) /\left(\left.\bar{q}\left(x_{1}\right)\right|_{1}\right)$ for $A=C, C a, F e$, and W. ns a function of $x_{1}$. Insert o for the Fe/D ratio shows the predietions of three models, discussed in the text. Insert d for the W/D ratio Hlso shows the $F_{2}(x)$ ratios inensured in DIS. 
to meson exchange. Various versions of the quark cluster models [10] also predicted a relative enhancement of the sea in heavier nuclei. The data appear consistent with the $Q^{2}$ rescaling argurr onts [11], though these models do not of course account for the "shadowing" effect at $x \leq 0.1$. Thus, the data gathered in this experiment support the following conclusions:

1 There is no evidence for the enhancement of sea quarks in the region $x \leq 0.3$.

2 The depletion seen in this DY ratio below $x=0.1$ is totally consistent with the observation seen in DIS. As the ratio of se: to valence is well below 0.5 in the region $0.05 \leq x \leq 0.1$, the valence quarks are "shadowed/depleted" to the same degree as the sea quarks. This is an interesting rew fact that awaits explanation.

\section{DILEPTON PAIRS FROM VECTOR MESONS}

Even though the gluonic sector carries roughly one-half of the proton's momentum at $Q^{2} \sim 20 \mathrm{GeV}^{2}$, there are very few direct measurements on this sector. No electroweak process directly couples to gluons, so that glue is generally viewed indirectly. There is some empirical evidence [12] that gluon fusion is principally respo:asible for the formation of heavy quark pairs $(c \bar{c}, b \bar{b}$, etc.). I $\mathrm{u}$ this experiment (E772), several $J^{\pi}=1^{-}$states of the $c \bar{c}$ and $b \bar{b}$ states are observed via their $\mu^{+} \mu^{-}$decay. I do not want to go into great detail on this subject, but want to share with you our results for this process as it bears both on the much-discussed issue of color transparency and production of these resonances in relativistic heavy-ion collisions.

Figures 4 and 5 clcarly show the yields for $J / \psi(1 s), \psi^{\prime}(2 s)$, and the $\Upsilon(1 s), \Upsilon^{\prime}(2 s)$, and $\Upsilon(3 s)$. Figure 9 shows the ratio of the $J / \psi$ yield for $\mathrm{C}, \mathrm{Fe}$, and $\mathrm{W}$ relative to $\mathrm{H}_{2}$ as a function of $x_{F}=x_{t}-x_{b}$. Clear evidence for an $A$ and $x_{F}$ dependerce is seen in this figure. The A dependence is summarized in Figure 6 , which shows the value of $\alpha$ obtained integrating over $x \boldsymbol{F}$ and assuming the yield is given by

$Y(A)=c A^{\mathbf{\alpha}}$.

Interestingly, the yield for $\psi^{\prime}$ shows the same A dependence as $J / \psi$. Calculations using wave functions that reproduce the $c \bar{c}$ energy spectrum show the $\psi^{\prime}$ to be twice as extended as the $\psi$. While it might be tempting to treat the A dependence as arising from an $A$ dependence of the gluon structure function, Figure 10 shows this to be unlikely. It shows the ratio for $J / \psi^{\prime}$ production from $F_{c} / D$ by incident protons at $E_{p}=200 \mathrm{GcV}$ [12] and our data at $E_{p}=800 \mathrm{GeV}$. Figure $10($ a) shows the two ratios as a function of $x_{F} \equiv x_{b}-x_{t}$, wherein beth experiments appear similar. However, when this ratio is converted to a function of $x_{t}$, it is clenr that the two mensurements do not yicld a common target structure function. The relative gluonic structure functions extracted from NA10 [13] end this experiment nre very cifferent. Progress is required if the yicld 


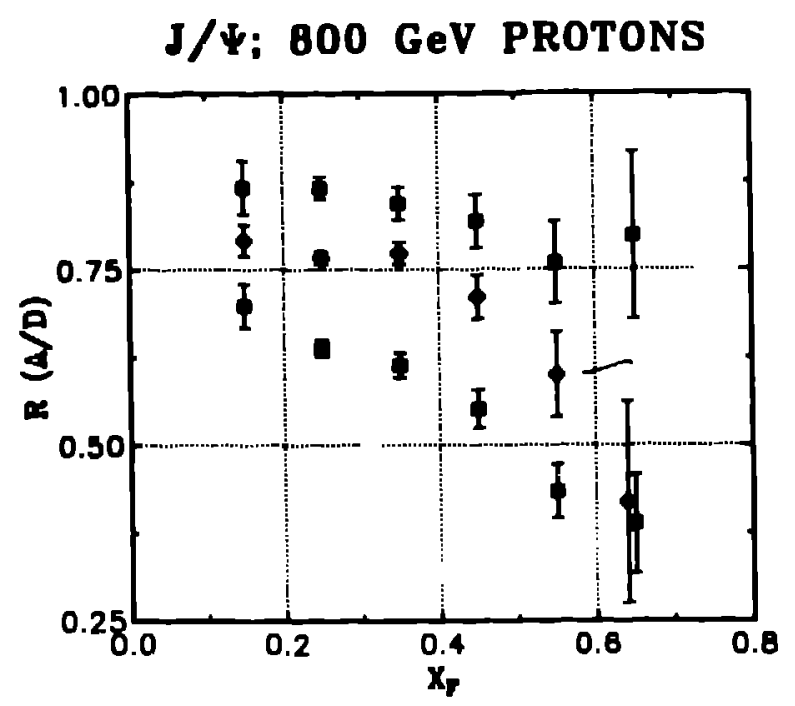

Figure 9. The ratio of $J / \psi$ production per nucleon for $\mathrm{C}$ (squares), Fe (diamonds), and $W$ (squares), relative to $D$. The ratio is shown as a function of $x_{F} \equiv x_{b}-x_{t}$.
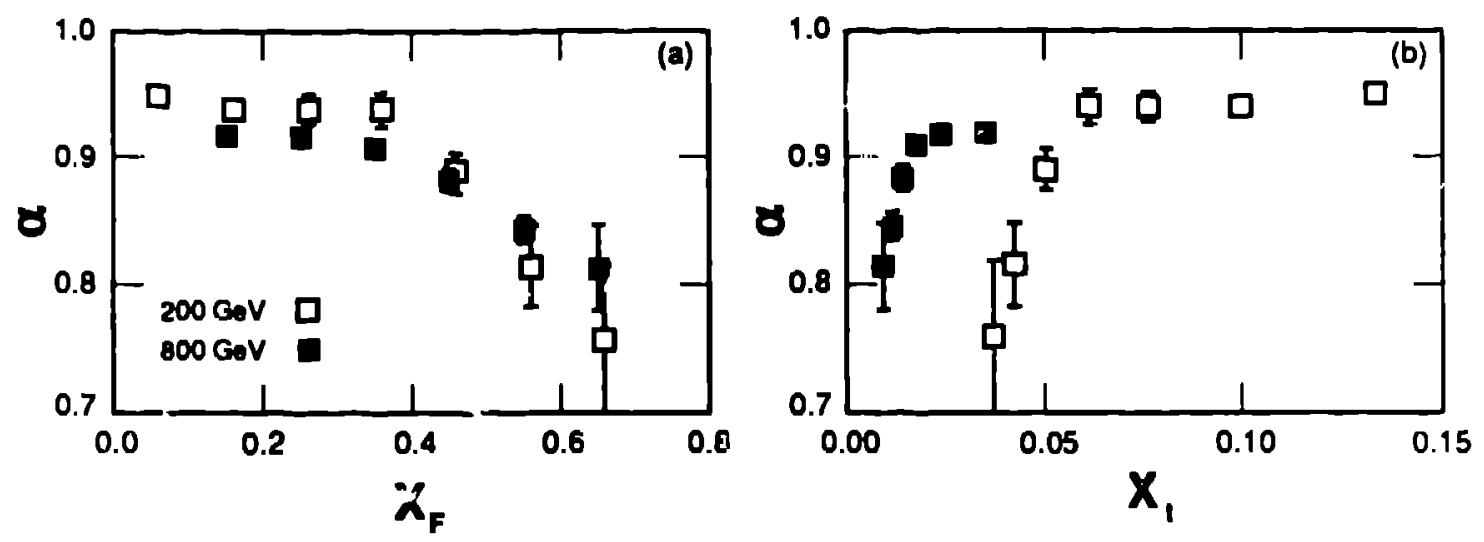

Figure 10. (a) The ratio of $J / \psi$ production per nucleon for Fe/D as a function of $x F$ for $E_{p}=200 \mathrm{GeV}$ (open squares) and $800 \mathrm{GeV}$ (solid squares). Note that the ratio is speciñed by the quantity $\alpha$ where $\alpha-1=\left[\ln \left(Y_{\text {Fe }} / Y_{D}\right)\right] / 3.25$. (b) Same ratio as $10(a)$, but now as a function of $x_{1}$.

of heavy-quark pairs is to be used as a measure of the turget and beam gluonic struiture functions.

The higher mass of the $\boldsymbol{Y}$ resonnnces makes the application of QCD casier and more reliable; therefore, one might believe that gluonic structure functions could be more reasonably extracted from the upsilon yicld. The $x \cdot$ distribution for the ratio of 
the $\Upsilon$ yield of W/D is show' $n$ in Figure 11(a), while the corresponding distribution is shown in Figure 11(b) as a function of $x_{t}$.

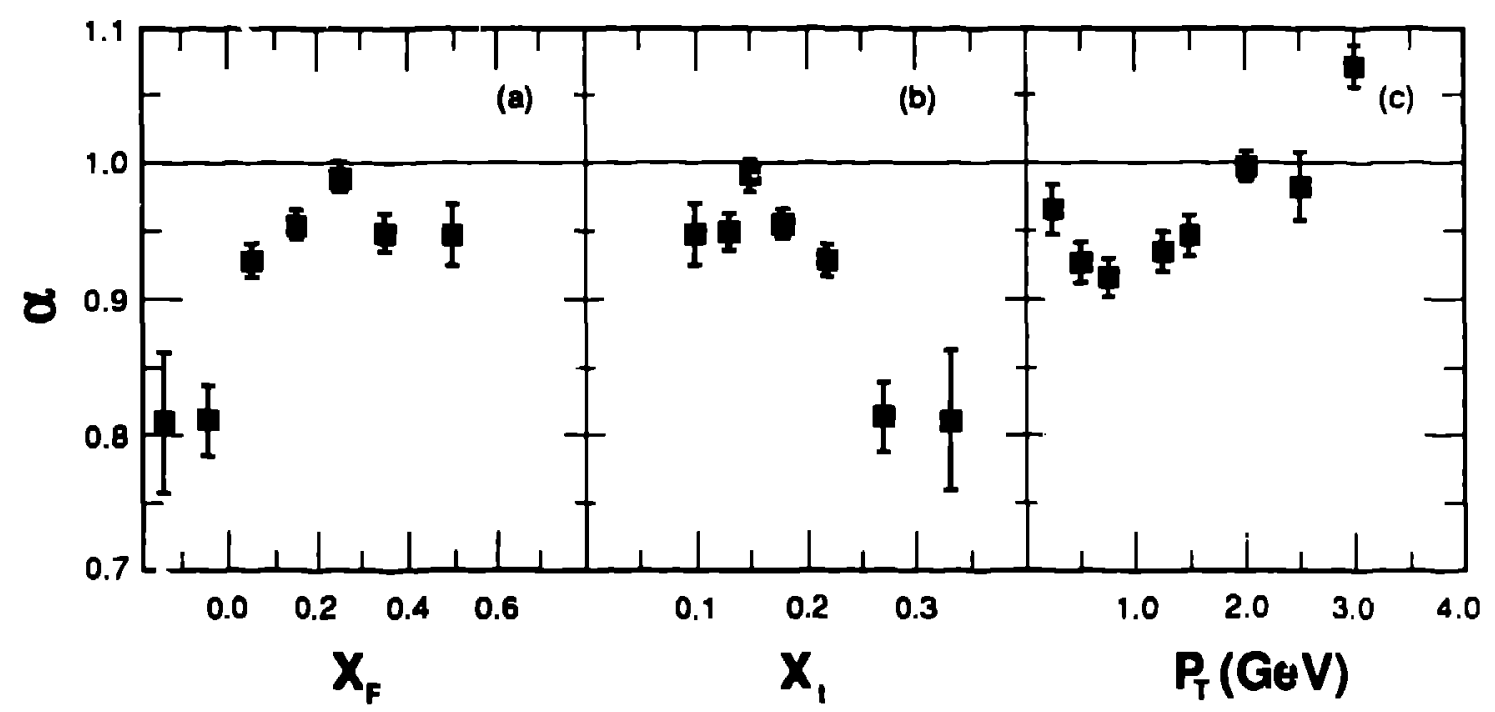

Figure 11. (a) The ratio of $\Upsilon(1 s)$ prodiction per nucleon for $W / D$. The ratio as a function of $x_{F}$ is given by the quantity $\alpha^{\prime}$ where $\alpha^{\prime}-1=\left[\ln \left(Y_{W} / Y_{D}\right)\right] / 4.52$. (b) Serne ratio as 11(a), but as a function of $x_{\imath}$. (c) Same ratio as 10(a), but as a function of the transverse momentum carried by the $\mu^{+} \mu^{-}$pair.

It appears that there must be considerable nuclear medium effects associated with the $J / \psi$ and $\Upsilon$ yields. If that is not the case, the very large $A$ dependence would require very depleted gluon structure functions in nuclei. This large depletion of glue would in turn present a severe problem for the momentum sum rule. Fortunately, the data do not support their use for the extraction of gluon structure functions as there is no consistent result from verious data sets.

\section{COMMENTS AND CLOSING REMARKS}

Before closing, allow me to make a few general observations on where this ares of research may be heading and the role of a ligh-energy ( $E \geq 20-\mathrm{GeV}$ ) CW electron accelerator. It will be necessary to use the Drell-Yun process to extend the A dependence of $\bar{q}(x)$ out to $x \sim 0.6$ with good statisties. If sea quarks arr in nucleons, then they should slow the effects clue to Fermi motion. Brrause the sen quark distribution: falls rapirlly, it shows enliancement dur to Fermi motion at appreciably suabler values of $x_{t}\left(x_{t} \sim 0.4\right)$ [14]. To pursue the A depenelenre of $\bar{q}(r)$ to much smaller values of 
$x_{\text {\& }}$ will require using Drell-Yan pairs with masses below the $J / \psi(3.1 \mathrm{GeV})$. Currently, only pairs with mass greater than $4 \mathrm{GeV}$ have been used. With the constraint,

$$
\frac{M_{\min }^{2}}{2 M_{p} t_{\ell}}<x_{b} x_{t}
$$

it is readily seen that to achieve $x_{t} \sim 10^{-3}$, either a collider must be used or the results extracted from using low-mass dilepton pairs. There may be an acceptable window near $M=2 \mathrm{GeV}$ hut the possibility that higher twist corrections at such a $Q^{2}$ might obscure the results needs to be investigated.

To further effectively pursue the EMC effect probably requires determination of the A dependence of flavor-specific structure functions. In particular, it would be most significant to obtain the A-dependence of the gluon structure function; however, there does not exist any clear experimental program that would achieve this. Neutrino DIS and Drell-Yan experiments have the possibility of obtaining flavor-specific quark structure functions. If electro-DIS is to further contribute to elaborating on the EMC effect, it will be via coincidence experiments that effectively tag the struck quark. Given our present understanding, this will be at best a statistical tagging and hence there will be considerable difficulty extracting distribut:ons that are smaller than, say, $10 \%$ of the dominant yield.

The polarizatior that can be readily achireved with a $\mathrm{CW}$ electron beam can potentially be an important factor, but it will require polarized targets of sufficient mass and resilience to carry out meaningful experiments. It also nppears thai even with the most ideal polarizec target, the experiments will be linited to very light. nuclei $(A<14)$ because, $\therefore$ ast. two (in odd-odal nuclei) nucleons can be aligned vin nuclear polarization. Thus, $i$ is not clear whrese a high-energy $\mathrm{CW}$ electron facility will :upart the A dependence observed in DIS.

There ure, of course, n host of other issues to be studied. Higher twist is a difficult pursuit and would be an extremely important element in the progrnm of proceeding from QCD to hadronir and nuclear physies. As much of higher twist. has to do with quark-quark corielations, it conceptunlly has much in rommon with the ever evasive

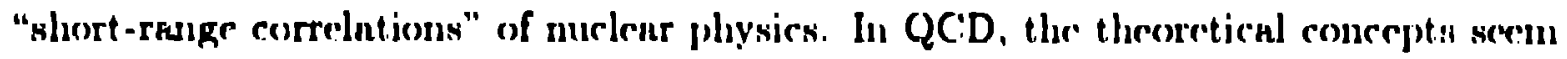
quite clens but complex. With no real examples of higher lwist from DIS experiments to work with, little progress has beren mode, hut the fut ure could be brighter.

'The study of hadronizntion, alomg with color transpareney. would doubtless ben." efit creatly from such on clectron fncility. The issue here in an evaluation of the

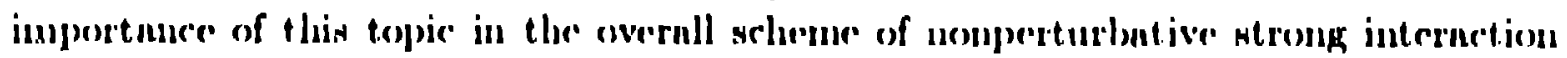
playsice nud the ontline of a set of experiment s thet would resolve the principul issues.

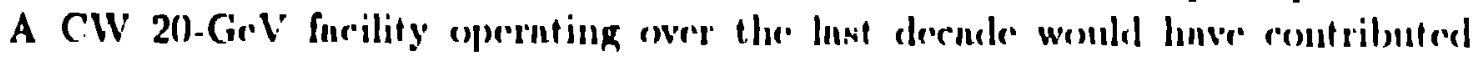

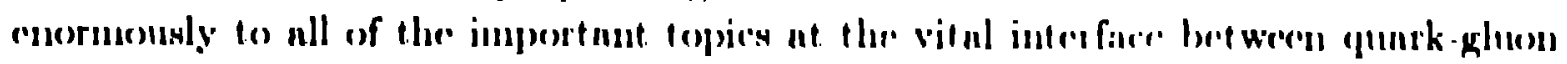

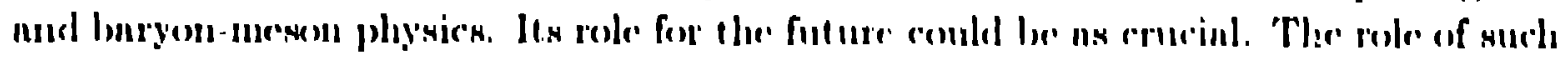


a facility must be investigated most energetically and expeditiously, so as not to be postponed beyond a time where ils impact could be decisive.

\section{REFERENCES}

1 J. J. Aubert et al., Phys. Lett. 123B (1983) 275.

2 U. Landgraff, in Proc. of the 12th International Conference on Particles and Nuclei (PANIC XII), Cambridge, Massachusetts, June 25-29, 1990 (to be published).

3 E772, "Precision A-Dependence of Dimuon Production at $800 \mathrm{GeV} / c$," M. R. Adams, D. M. Alde, H. W. Baer, B. L. Barlett, C. N. Brown, T. A. Carey, W. E. Cooper, G. Danner, G. T. Garvey, R. Guo, Y. B. Hsiung, G. W. Hoffman, D. M. Kaplan, A. Klein, C. Lee, M. J. Leitch, J. W. Lillberg, R. L. McCarthy, P. L. McGaughey, C. S. Mishra, J. M. Moss, J. C. Peng, and M. J. Wang.

4 S. D. Drell and T. M. Yan, Phys. Rev. Lett. 25 (1970) 316.

5 M. Ericson and A. W. Thomas, Phys. Lett. 128B (1983) 112; E. L. Berger, F. Coester, and R. B. Wringa, Phys. Rev. D29 (1984) 398.

6 R. P. Bickerstaff et al., Phys. Rev. Lett. 53 (1984) 2531; M. Ericson and A. W. Thomas, Phys. Lett. 148B (1984) 191; E. L. Berger, Nucl. Phys. B267 (1986) 231.

7 J. A. Crittenden et al., Phys. Rev. D34 (1986) 2584.

8 G. 1'. Bodwin, S. J. Brodsły, and G. P. Lepage, Phys. Rev. Lett. 47 (1981) 1799; A. H. Mueller, in Proc. of the 1985 International Symposium on Lepton and Photon Interbctions at High Energies, Kyoto, Japan.

9 H. Abramowicz et al., Z. Phys. C25 (1984) 29.

10 C. E. Carlson and T. J. Havens, Phys. Rev. Lett. 51 (1983) 201.

11 F. E. Close, R. L. Jaffe, R. G. Roberts, and G. G. Ross, Phys. lic.v. D31 (1085) 1004.

12 J. Badius et al., Z. Phys. C20 (1083) 101.

13 A. D. Martin, R. G. Roberts, and W. J. Stirling, Phys. Rev. D3i (1988) 1101.

14 R. L. Jaffe, in Relativistic Dynamirs and Quark-Nucleon Physics, M. B. Johnson and A. Picklesimer, Eds. (Join Wilcy und Sons, New York, 1986). 